\title{
Potentiel hydrique et conductance stomatique des feuilles de frêne (Fraxinus excelsior $L$ ) dans une forêt alluviale du Haut-Rhône français
}

\author{
G Besnard, G Carlier* \\ Laboratoire de botanique et biologie végétale, université Joseph Fourier (Grenoble 1), \\ 38041 Grenoble Cedex, France
}

(Reçu le 28 avril 1989; accepté le 18 janvier 1990)

\begin{abstract}
Résumé - L'article décrit l'évolution de l'intensité de la transpiration, de la conductance stomatique et du potentiel hydrique des feuilles de frêne au cours de plusieurs journées d'été, dans une forêt alluviale du Haut-Rhône français. Les relations entre ces grandeurs et l'influence des facteurs micrométéorologiques sont discutées. Durant les étés 1986 et 1987. le potentiel de l'eau du sol, dans la zone des racines, n'a jamais été inférieur à $-0,025 \mathrm{MPa}$, sauf à $25 \mathrm{~cm}$ de profondeur où il a atteint temporairement $-0,1 \mathrm{MPa}$. La conductance stomatique dépend principalement de l'éclairement; par temps ensoleillé, elle reste pendant une grande partie de la journée au voisinage de sa valeur maximale $\left(16 \times 10^{-3} \mathrm{~m} \cdot \mathrm{s}^{-1}\right)$. La conductance maximale est remarquablement élevée par rapport à celles d'autres arbres de régions tempérées, ce qui permet une transpiration intense justifiant la réputation du frêne d'être un gros consommateur d'eau. Le potentiel hydrique foliaire est corrélé très significativement avec la transpiration; il décroît très rapidement dans la matinée; si la transpiration est intense, le potentiel minimal peut atteindre $-1,94 \mathrm{MPa}$ (moyenne pour 5 feuilles). En aucune circonstance, la chute diurne du potentiel hydrique foliaire n'a provoqué de fermeture stomatique, ce qui s'accorde bien avec la forte productivité des frênaies alluviales. En revanche, une régulation stomatique reste possible en cas de chute plus importante du potentiel hydrique foliaire, comme le montre l'évolution de la conductance et du potentiel des feuilles d'une branche coupée.
\end{abstract}

frêne / conductance stomatique / transpiration / potentiel hydrique foliaire / forêt alluviale tempérée

Summary - Water potential and stomatal conductance of ash (Fraxinus excelsior $\mathrm{L}$ ) leaves in an alluvial forest of the French upper Rhone valley. This paper reports on the timecourse of the transpiration rate, stomatal conductance and water potential of ash leaves during selected summer days in an alluvial forest of the French upper Rhone valley. The relation between these physiological quantities and the influences exerted on them by the micro-

* Correspondance et tirés à part 
meteorological factors are discussed. During the summers of 1986 and 1987, the depth of the water table at the research site remained close to $1.90 \mathrm{~m}$. The soil water potential in the root zone never fell below -0.025 MPa except at $25 \mathrm{~cm}$ depth where it temporarily attained $-0.1 \mathrm{MPa}$. The stomatal conductance depends mainly on the light intensity. On sunny days, the stomatal conductance tends to remain close to its maximum value $\left(16 \times 10^{-3-}\right.$ $\mathrm{m} \cdot \mathrm{s}^{-1}$ ) for most of the day. This maximal value is higher than that of many other temperate trees, thus allowing a high transpiration rate. Ash's reputation for high water consumption therefore appears justified. The leaf water potential is highly correlated with the transpiration rate. It decreases sharply in the morning and may fall as low as $-1.94 \mathrm{MPa}$ when the transpiration rate is high. These such low water potentials do not appear to induce the closure of stomata in any case, which agrees with the known high productivity of ash in alluvial communities. On the other hand, stomatal regulation seems to occur with a further decrease in leaf water potential, as is suggested by the time course of conductance and potential in the leaves of a cut branch.

\section{ash / stomatal conductance / transpiration / leaf water potential / temperature allu- vial forest}

\section{INTRODUCTION}

Dans la plaine alluviale du Rhône entre Genève et Lyon, on observe depuis un siecle une extension croissante de frênaies à Fraxinus excelsior L. Ces frênaies s'installent à la suite des groupements mésohygrophiles à Alnus incana (L) Moench ou à Alnus glutinosa L lorsque la nappe phréatique s'abaisse au-dessous de 1,5 à $2 \mathrm{~m}$ (Richard et Pautou, 1982). Dans la zone où se trouve notre site d'étude, l'abaissement du niveau de la nappe par rapport a la surface du sol résulte :

- de l'alluvionnement occasionné par les crues et;

- de la dérivation d'une partie du débit du fleuve vers le canal d'alimentation d'une usine hydroélectrique.

Le frêne est une espèce de lumière, craignant les gelées de printemps, à forte préférence pour les sols bien pourvus en eau, ni trop tassés, ni trop argileux, mais défavorisée par la présence d'une nappe superficielle; ses racines doivent pouvoir s'enfoncer jusqu'à $1 \mathrm{~m}$ ou, mieux 1,5 m (Devauchelle et Lévy, 1977; Le Goff et Lévy, 1984). Le frêne contribue à la forte productivité des fo- rêts alluviales de bois durs (Pautou et Decamps, 1985), mais il se trouve aussi, associé au hêtre, à l'étage montagnard (Richard et Pautou, 1982).

Le frêne est un gros consommateur d'eau (Ladefoged, 1963 ; Braun, 1977). Aussenac et Lévy (1983), grâce à des expériences sur sujets de 2 ans en cuves de végétation, expliquent sa localisation préférentielle dans les biotopes à bonne alimentation en eau par le déclenchement tardif de la régulation stomatique en cas de détresse hydrique.

Le travail décrit ici a pour objet de recueillir des informations sur le comportement stomatique et le potentiel hydrique foliaire du frêne dans les conditions naturelles d'une zone alluviale, durant la période estivale, afin de contribuer à expliquer sa localisation écologique (Aussenac, 1985).

\section{MATÉRIEL ET MÉTHODES}

\section{Localisation}

Le site d'étude est situé sur l'île des Molottes, commune des Avenieres, département de I'Isère, approximativement à égale 
distance de Lyon et Genève (45.35' N, $5^{\circ} 56^{\prime} \mathrm{E}$ ). L'altitude est de $207 \mathrm{~m}$. Le sol est de texture principalement sableuse avec, vers $2 \mathrm{~m}$ de profondeur, un horizon de graviers et cailloux. Le groupement végétal est principalement composé de Fraxinus excelsior $L$ et Alnus incana ( $L$ ) Moench pour les arbres, Evonymus europaea L, Cornus sanguinea $L$ et Crataegus monogyna Jacq pour les arbustes, Impatiens glandulifera Royle, Allium ursinum $\mathrm{L}$ et Rubus caesius $\mathrm{L}$ pour les herbacees. II s'agit d'un groupement de transition entre l'aulnaie à Alnus incana et la frênaie.

Les frênes ont une hauteur moyenne de $10 \mathrm{~m}$, un diamètre (à $1,30 \mathrm{~m}$ de hauteur) inférieur à $10 \mathrm{~cm}$ et recouvrent environ $25 \%$ de la surface du sol. L'enracinement est dense jusque vers $0,25 \mathrm{~m}$ et reste notable jusqu'à $1,10 \mathrm{~m}$; quelques racines atteignent $1,50 \mathrm{~m}$.

Les résultats d'une recherche parallèle, effectuée dans la même station sur l'aulne blanc, ont été publiés (Besnard et Carlier, 1988).

\section{Méthodes}

\section{Mesures physiologiques}

Les mesures sont faites sur des feuilles adultes du sommet des houppiers de 4 arbres accessibles à partir d'une plateforme.

Le potentiel hydrique foliaire $\psi f$ est mesuré à l'aide d'une chambre de pression (Scholander et al, 1965) réalisée par Collicard (1982). Pour limiter l'évaporation se produisant après l'excision de la feuille, la mesure est faite dans les 2 min qui suivent, et la chambre est tapissée d'un papier filtre imbibé d'eau distillee.

La transpiration TR et la résistance stomatique à la diffusion de vapeur d'eau $R D$ sont mesurées à l'aide d'un poromètre à régime stationnaire Li-Cor 1600. Cet appareil n'est pas utilisable lorsque les feuilles sont mouillées par la rosée ou lorsque l'humidité de l'air est supérieure à $85 \%$. La mesure est faite sur la face abaxiale. En effet, un essai préliminaire a montré que, lorsque les stomates sont ouverts, la résistance de la face adaxiale est environ 25 fois plus élevée que celle de l'autre face. La résistance d'ensemble de la feuille, calculée selon Pallardy et Kozlowski (1981) est donc pratiquement égale à celle de la face abaxiale. Pour la présentation des résultats, on calcule la conductance stomatique, qui est l'inverse de la resistance.

La mesure au porometre est effectuée la première, sur une feuille en place; celle-ci est aussitôt cueillie pour la mesure du potentiel hydrique. Cinq feuilles sont traitees successivement. Les mesures sont répétées 6 a 9 fois au cours de la journée.

Les heures sont indiquées en temps universel (TU), en retard de $30 \mathrm{~min}$ sur l'heure solaire locale.

Une expérience, destinée à préciser les rapports entre conductance stomatique et potentiel hydrique foliaire, a consisté à suivre l'évolution de ces 2 grandeurs au cours de la perte d'eau par les feuilles portées par une branche coupée, méthode utilisée par Beadle et al, 1978. Les conditions microclimatiques réalisées au cours de cette expérience sont précisées plus loin (section Resuitats).

\section{Mesures microclimatiques}

Un capteur Li-Cor 190 SB, sensible aux photons de longueur d'onde comprise entre 400 et $700 \mathrm{~nm}$, est installé sur le poromètre. La mesure de la transpiration et de la résistance stomatique de chaque feuille est donc accompagnée de celle du flux de photons (éclairement) perpendiculaire au limbe in situ.

La température de l'air $t$ et son humidité relative $H R$ sont mesurees à l'aide d'un psychrometre à ventilation $\mathrm{J}$ Richard.

\section{Mesures concernant l'eau du sol}

La profondeur de la nappe est repérée grâce à un piézomètre. La charge hydraulique $H$ du sol $(H<0)$ est lue sur des tensiomètres Nardeux DTM 5000 enfoncés aux profondeurs $Z$ $(Z<0)$ égales à $25,65,105$ et $155 \mathrm{~cm}$ (Vachaud et al, 1978). Le potentiel hydrique $\psi_{s}$ est calculé d'après la relation :

$\psi_{s}(\mathrm{MPa})=H(\mathrm{MPa})+Z(\mathrm{~cm}) / 10000$ Lorsque la charge hydraulique tombe en dessous de $-0,08 \mathrm{MPa}$, de l'air peut s'introduire dans le tensiomètre et la lecture n'est plus valable. Ceci ne s'est produit que du 7 au 9 août 1986 et seulement pour le tensiomètre situé à $25 \mathrm{~cm}$ de profondeur. 


\section{RÉSULTATS}

\section{Évolution de l'état de l'eau dans le sol}

Pendant les étés 1986 et 1987, la profondeur de la nappe est restée comprise entre 1,75 et $1,95 \mathrm{~m}$, à l'exception d'une période d'inondation par crue du Rhône en juin 1987.

En profondeur, le sol reste toujours proche de la saturation : au-delà de $1 \mathrm{~m}$ de profondeur, la charge hydraulique est toujours supérieure à $-0,019 \mathrm{MPa}$ et varie très peu. Par contre, les horizons supérieurs sont soumis à l'extraction par les racines et a l'évaporation superficielle : la charge hydraulique y a atteint un minimum de $-0,1 \mathrm{MPa}$ à $25 \mathrm{~cm}$ de profondeur, mais $-0,031 \mathrm{MPa}$ seulement à $65 \mathrm{~cm}$, durant la première décade d'août 1986. Cet assèchement relatif a fait suite à l'absence de précipitations du 7 juillet au 10 août 1986 . Le 11 août 1986, un orage a apporté $60 \mathrm{~mm}$ d'eau; les précipitations ont été ensuite régulièrement réparties jusqu'à la fin de l'été 1986 (80 à $100 \mathrm{~mm}$ par mois) et la charge hydraulique n'est plus descendues au-dessous de $-0,02 \mathrm{MPa}$, à aucune profondeur. Une représentation détaillée de ces données a déjà été publiée (Besnard et Carlier, 1988).
Après un mois de juin très pluvieux (plus de $200 \mathrm{~mm}$ d'eau) l'été $1987 \mathrm{a}$ comporté des précipitations régulières et la charge hydraulique n'a jamais été inférieure à $-0,035 \mathrm{MPa}$ à $25 \mathrm{~cm}$ de profondeur.

\section{Évolution journalière des grandeurs physiologiques}

Les conditions micrométéorologiques réalisées sur le site au cours de 6 journées de mesure sont résumées sur le tableau $I$.

$A$ titre d'exemple, les figures 1 et 2 présentent le détail de l'évolution des grandeurs micrométéorologiques et physiologiques au cours de 2 journées.

\section{La conductance stomatique et la transpiration}

En raison de la présence régulière de rosée, la conductance stomatique ne peut être mesurée tôt le matin. II n'est donc pas possible de percevoir le début de la phase d'ouverture des stomates.

Lorsqu'on peut réaliser au moins 2 mesures, on constate une augmentation de la conductance concomitante à celle de l'éclairement, jusque vers

Tableau I. Résumé des conditions micrométéorologiques des journées de mesures.

\begin{tabular}{|c|c|c|c|}
\hline Ensoleillement & $\begin{array}{c}\text { Temperature } \\
\text { maximale } \\
{ }^{\circ} \mathrm{C}\end{array}$ & $\begin{array}{l}\text { Humidité } \\
\text { relative } \\
\text { minimale \% }\end{array}$ & $\underset{\substack{D P V \\
\text { maximal }}}{\stackrel{D P a}{ }}$ \\
\hline $\begin{array}{l}8 \text { juill } 86 \text { bon, sauf mi-journée } \\
21 \text { juill } 86 \text { continu } \\
30 \text { juill } 86 \text { bon, sauf mi-journée } \\
6 \text { août } 86 \text { continu } \\
12 \text { sept } 86 \text { médiocre, pluie à } 16 \text { h } \\
27 \text { sept } 86 \text { bon } \\
27 \text { juill } 87 \text { bon, sauf mi-journée }\end{array}$ & $\begin{array}{l}23 \\
25 \\
30 \\
26 \\
22 \\
20,4 \\
22\end{array}$ & $\begin{array}{l}66 \\
57 \\
55 \\
59 \\
65 \\
85 \\
50\end{array}$ & $\begin{array}{l}0,9 \\
1,4 \\
1,8 \\
1,4 \\
0,8 \\
0,4 \\
1,3\end{array}$ \\
\hline
\end{tabular}



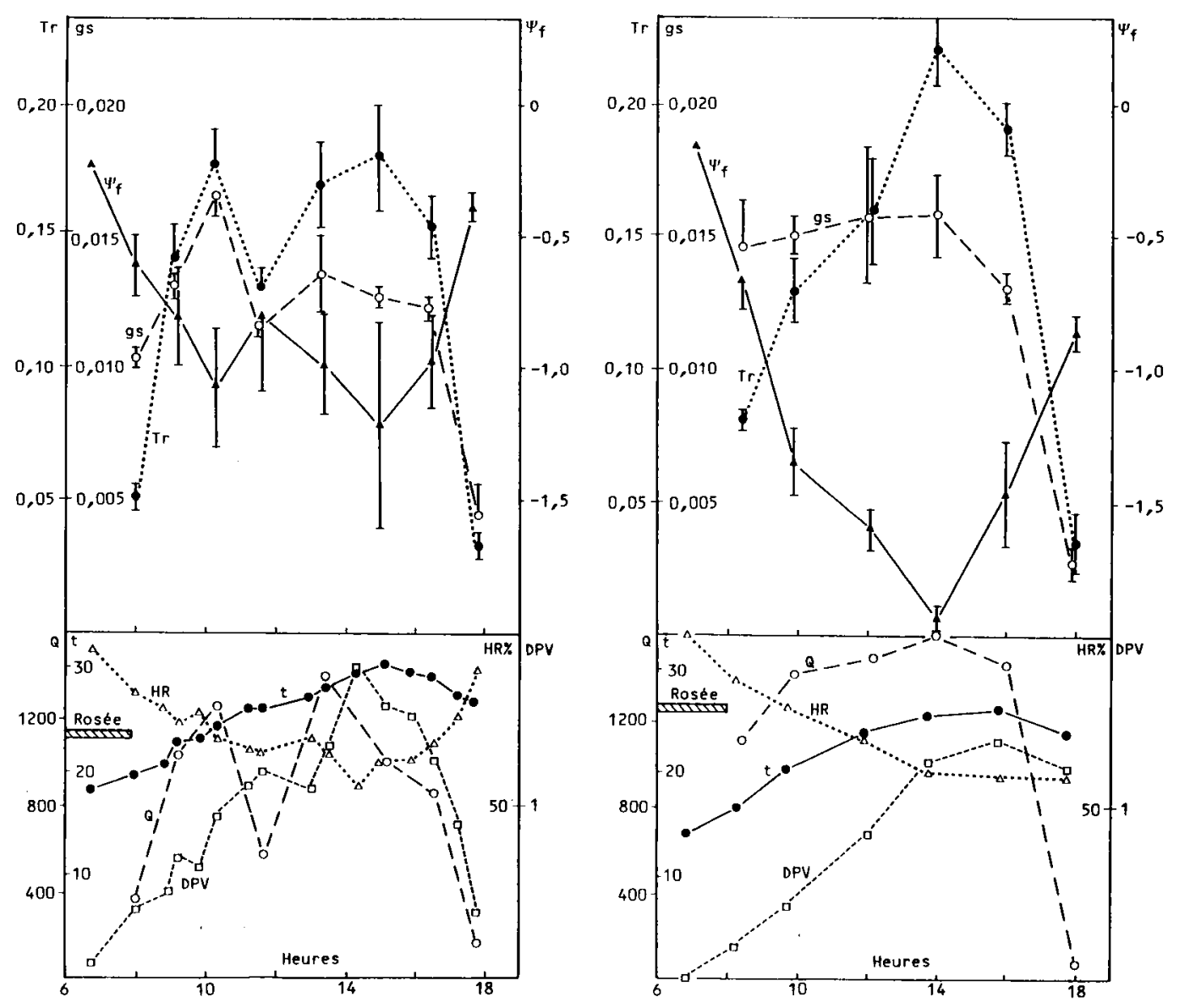

Figs 1 et 2. Évolution des grandeurs micrométéorologiques (en bas) et physiologiques (en haut) pendant les journées du 30 juillet 1986 (fig 1, à gauche) et du 6 août 1986 (fig 2, à droite). Tr = transpiration $\left(\mathrm{g} \cdot \mathrm{s}^{-1} \cdot \mathrm{m}^{-2}\right) \cdot \psi_{f}=$ potentiel hydrique foliaire $(\mathrm{MPa}) . g_{s}=$ conductance stomatique pour la diffusion de vapeur d'eau $\left(\mathrm{m} \cdot \mathrm{s}^{-1}\right) . t=$ température de l'air $\left({ }^{\circ} \mathrm{C}\right) . H R=$ humidité relative de l'air $(\%)$. DPV = déficit de pression de vapeur d'eau de l'air $(\mathrm{kPa}), Q_{2}=$ éclairement ou densité de flux des radiations actives sur la photosynthese ( $\mu$ moles (photons) $\mathrm{m}^{-2} \cdot \mathrm{s}^{-1}$ ). Heures en TU. Les barres représentent l'écart type.

$10 \mathrm{~h} 30$, heure à laquelle la conductance a généralement atteint sa valeur maximale (Tableau 11). Si l'ensoleillement est permanent, comme le 6 août (fig 2), la conductance ne varie pas significativement jusque vers $16 \mathrm{~h} 00$. En cas de passage nuageux (fig 1), la conductance subit des variations dont le sens (mais pas forcément l'amplitude) coïncide bien avec les oscillations de l'éclairement. La phase de fermeture des stomates en fin d'après-midi correspond à la diminution de l'éclairement. Le maximum absolu de toutes les valeurs individuelles disponibles de conductance stomatique atteint $20 \times 10^{-3} \mathrm{~m} \cdot \mathrm{s}^{-1}$, mais la moyenne pour 5 feuilles ne dépasse pas $16,4 \times 10^{-3} \mathrm{~m} \cdot \mathrm{s}^{-1}$ (tableau II).

La transpiration varie dans le même sens que la conductance stomatique le 
Tableau II. Valeurs maximales de la conductance stomatique $g_{s}$ et de la transpiration $T r$ et valeurs minimales du potentiel hydrique foliaire $\psi_{t}$ (moyennes \pm écarts types).

\begin{tabular}{|c|c|c|c|c|c|c|c|}
\hline \multirow[b]{2}{*}{ Dates } & \multirow[b]{2}{*}{$\begin{array}{c}\mathrm{g}_{\mathrm{s}} \operatorname{maxi} \\
m \cdot \mathrm{s}^{-1} \times 1000\end{array}$} & \multirow[b]{2}{*}{$\begin{array}{c}\operatorname{Tr} \operatorname{maxi} \\
g \cdot s^{-1} \cdot m^{-2}\end{array}$} & \multirow[b]{2}{*}{$\begin{array}{c}\psi f \operatorname{mini} \\
\mathrm{MPa}\end{array}$} & \multirow[b]{2}{*}{ Heures } & \multicolumn{3}{|c|}{$\begin{array}{c}\text { Conditions aux mêmes } \\
\text { moments }\end{array}$} \\
\hline & & & & & $\begin{array}{c}Q \\
\mu \mathrm{mol} \cdot \mathrm{m}^{-2} \cdot \mathrm{s}^{-1}\end{array}$ & ${ }^{\circ} \mathrm{C}$ & $\begin{array}{l}D P V \\
k P a\end{array}$ \\
\hline 8 juill 86 & $15,9 \pm 1,3$ & $0,142 \pm 0,010$ & & 10.10 & 1590 & 20,5 & 0,70 \\
\hline & & & $-1,10 \pm 0,06$ & 16,15 & 873 & 20,7 & $\begin{array}{l}0,85 \\
0,50\end{array}$ \\
\hline 21 JuII 86 & $14,7 \pm 1,3$ & $0,169 \pm 0,022$ & $-1,12 \pm 0,19$ & $\begin{array}{r}8,30 \\
11,00\end{array}$ & 1590 & 20 & 0,87 \\
\hline 30 juill 86 & $16,4 \pm 1,1$ & & & 10,30 & 1260 & 24,5 & 0,94 \\
\hline 6 août 86 & $16,4 \pm 1,3$ & $0,182 \pm 0,021$ & $-1,22 \pm 0,40$ & $\begin{array}{l}15,00 \\
10,10\end{array}$ & $\begin{array}{l}1000 \\
1440\end{array}$ & $\begin{array}{l}30,2 \\
20,6\end{array}$ & $\begin{array}{l}1,59 \\
0,44\end{array}$ \\
\hline & & $0,223 \pm 0,017$ & $-1,94 \pm 0,06$ & 14,10 & 1600 & 25,7 & 1,26 \\
\hline 12 sept 86 & $15,9 \pm 1,0$ & $0,118 \pm 0,007$ & $-1,00 \pm 0,14$ & 10,35 & 990 & 19,7 & 0,36 \\
\hline 27 sept 86 & $12,2 \pm 8,7$ & $0,101 \pm 0,041$ & $-0,96 \pm 0,34$ & 14,35 & 1340 & 20 & 0,35 \\
\hline 27 juill 87 & $11,9 \pm 0,9$ & $0,108 \pm 0,004$ & $-1,08 \pm 0,36$ & 10,30 & 1660 & 20,4 & 1,10 \\
\hline
\end{tabular}

matin, le soir, et en cas de passage nuageux (fig 1). Mais, pendant les jours les plus chauds, où le DPV est aussi le plus élevé, le maximum de la transpiration est atteint plus tard que celui de la conductance (tableau II).

\section{Le potentiel hydrique foliaire}

Le potentiel de base, mesuré alors que les feuilles sont encore recouvertes de rosée, est compris entre $-0,08$ et $-0,2 \mathrm{MPa}$. La corrélation entre le potentiel de base et le potentiel de l'eau du sol, même dans la couche la plus superficielle $(25 \mathrm{~cm})$, n'est pas significative (tableau III). L'écart moyen entre potentiel de base et potentiel du sol dans cette couche est voisin de 0,1 $\mathrm{MPa}$, ce qui correspond bien à la dénivellation de $10 \mathrm{~m}$ entre sol et feuilles.

Le potentiel chute rapidement jusque vers $10 \mathrm{~h} 00$. Ensuite, suivant les jours, il peut se maintenir à peu près constant durant la journée, remonter légèrement dans l'après-midi, ou encore continuer à décroître. Comme on pouvait s'y attendre, les variations du po-

Tableau III. Comparaison du potentiel de base $\psi_{b}$ et du $\psi$ du sol (MPa)

\begin{tabular}{rllll}
\hline \multicolumn{1}{c}{ Dates } & $\begin{array}{c}\psi_{b} \\
\text { moyen }\end{array}$ & à $25 \mathrm{~cm}$ & à $65 \mathrm{~cm}$ & $\Delta \psi^{*}$ \\
\hline 8 juill 86 & $-0,1$ & & & \\
22 juill 86 & $-0,1$ & $-0,024$ & $-0,014$ & $-0,076$ \\
30 juill 86 & $-0,2$ & $-0,045$ & $-0,019$ & $-0,155$ \\
6 août 86 & $-0,15$ & $-0,062$ & $-0,021$ & $-0,088$ \\
12 sept 86 & $-0,08$ & $-0,016$ & $-0,013$ & $-0,064$ \\
23 sept 86 & $-0,09$ & $-0,008$ & $-0,011$ & $-0,082$ \\
27 sept 87 & & $-0,009$ & $-0,008$ & \\
\hline
\end{tabular}

${ }^{*} \Delta \psi=\psi_{b}-\psi$ du sol à $25 \mathrm{~cm}$ Corrélation entre $\psi_{b}$ et $\psi$ du sol à $25 \mathrm{~cm}: r=0,79 \mathrm{NS}$; Corrélation entre $\psi_{b}$ et $\psi$ du sol à $65 \mathrm{~cm}: r=0,84 \mathrm{NS}$. 
tentiel sont inverses de celles de la transpiration. Le minimum de potentiel coïncide à une exception près (le 8 juillet 86 ) avec le maximum de la transpiration (tableau II) et, pour l'ensemble de toutes les mesures disponibles, le minimum absolu du potentiel coïncide avec le maximum absolu de la transpiration (le 6 août 86 ).

La fin de journée est toujours marquée par une remontée très nette du potentiel, mais les mesures n'ont jamais pu être prolongées suffisamment tard pour suivre le retour au potentiel de base mesuré le matin.

\section{Relations entre les grandeurs mesurées}

\section{Relations entre la conductance stomati- que $\boldsymbol{g}_{s}$ et les facteurs microclimatiques.}

La relation entre la conductance et l'éclairement reçu $Q$ peut être mise sous la forme proposée par Burrows et Milthorpe (1976) :

$$
1 / g_{s}=(a+b) / Q
$$

Si l'on prend en compte la totalité des mesures (157 couples) cette relation s'écrit :

$$
1 / g_{s}=73+16166 / Q
$$

indiquant que la conductance vaut la moitie de son maximum pour $Q=$ $16166 / 73$ soit $221 \mu \mathrm{mol} \cdot \mathrm{m}^{-2} \cdot \mathrm{s}^{-1}$. La corrélation est hautement significative $\left(r=0,77^{* *}\right)$. Si l'on considere les mesures journée par journée, les corrélations sont encore meilleures, sauf le 8 juil et le 23 sept 1986 où elles ne sont pas significatives; ces 2 jours-là, il n'y a pas eu de mesures sous faible éclairement. En fait, pour les éclairements superieurs à $700 \mu \mathrm{mol} \cdot \mathrm{m}^{-2} \cdot \mathrm{s}^{-1}$, les variations de conductance ne sont plus significatives : l'ouverture des stomates est maximale.
II n'existe pas de relation significative entre la conductance et les autres facteurs microclimatiques. On constate cependant que les valeurs les plus élevées de la conductance ont été observées aux températures au plus égales à $26{ }^{\circ} \mathrm{C}$ et aux $D P V$ au plus égaux à $1,3 \mathrm{kPa}$; il peut s'agir d'un effet unique car ces 2 variables sont en étroite corrélation. Il en résulte une réduction de la transpiration quand le temps est très chaud et sec.

\section{Relations entre le potentiel hydrique foliaire $\psi_{f}$ et la transpiration Tr.}

Le potentiel hydrique foliaire varie en sens inverse de la transpiration. Pour l'ensembie des données, il existe entre $\Psi f$ et $T r$ une relation linéaire du type proposé par Elfving et al (1972), qui s'écrit :

$$
\psi f=-0,32-5,14 \mathrm{Tr}
$$

La corrélation est hautement significative $\left(r=0,68^{\star \star}\right)$. La relation curviligne faisant intervenir un terme en $\pi r^{2}$ n'est pas plus significative. L'évolution dans le temps de $\psi_{f}$ et $\operatorname{Tr}$ (fig 3 ) ne montre pas d'effet d'hysteresis : à transpiration égale, il n'y a pas de différence systématique entre le $\psi f$ du matin et celui de l'aprèsmidi. La relation linéaire et l'absence d'hysteresis indiquent l'existence du flux d'eau conservatif (Katerji et al, 1983) postulé par le modèle du Van den Honert (Milburn, 1979) pour la circulation de l'eau dans le système sol- plante-atmosphère.

Suivant Pallardy et Kozlowski (1981) la pente de la partie linéaire de la relation $\psi f=f(T r)$ rend compte de la résistance au flux d'eau liquide atteignant les feuilles dont la transpiration est mesuree; l'inverse de la pente exprime la conductance hydraulique. Pour le frêne, d'après 


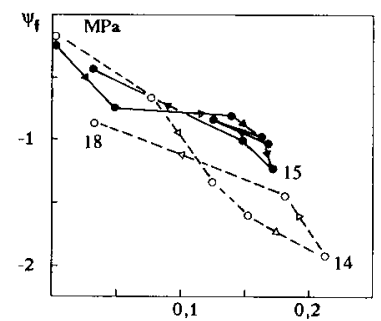

Fig 3. Évolution journalière de la relation entre la transpiration et le potentiel hydrique foliaire. Les courbes se lisent dans le sens des flèches. 30 juill 86 . $0: 6$ août 86

la relation numérique ci-dessus, cette conductance vaut $10,8 \times 10^{-9} \mathrm{~mol}$. $\mathrm{m}^{-2} \cdot \mathrm{s}^{-1} \cdot \mathrm{Pa}^{-1}$ (si l'on adopte le type d'unité employé par Schulze et al, 1985).

\section{Relations entre la conductance sto- matique $g_{s}$ et le potentiel $\psi f$}

II est important de savoir si, dans les conditions biotopiques du présent travail, un mécanisme stomatique limitant la chute du potentiel fonctionne chez le frêne. Un indice de l'intervention d'un tel mécanisme peut être fourni par la boucle reliant, dans l'ordre chronologique, les points définis par les couples $\left(\psi f-g_{s}\right)$. Par temps bien ensoleillé, il y a d'abord, en début de journée, augmentation de la conductance et chute du potentiel. Puis, dans le cas où une régulation stomatique intervient, la conductance diminue tandis que le potentiel reste constant. En fin de journée la conductance est devenue faible et le potentiel remonte (Körner et Cochrane, 1985).

Dans les cas étudiés ici, ce schéma (fig 4) est presque toujours compliqué par la réponse des stomates aux passages nuageux (exemple le 30 juillet 1986). Mais le 6 aout 1986, journée sans nuages, la conductance maximale est conservée durant plusieurs heures alors que $\psi f$ décroît jusqu'à la plus basse valeur mesurée dans toute l'étude $(-1,94 \mathrm{MPa})$. II n'intervient donc pas de régulation stomatique.

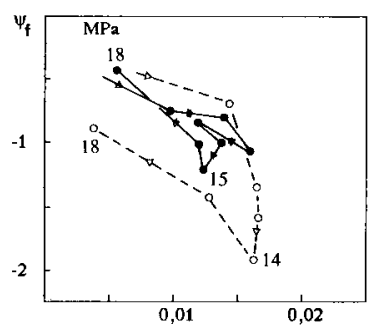

Fig 4. Évolution journalière de la relation entre la conductance stomatique et le potentiel hydrique foliaire. Les courbes se lisent dans le sens des flèches. : 30 juill 86 . $\circ: 6$ août 86 .

Pour déterminer si des conditions plus sévères peuvent causer une fermeture diurne des stomates, une branche a été coupée le 27 juillet 87 à 13 h et déposée au sol. Par rapport aux conditions régnant au niveau des houppiers, cette branche a été soumise à des temperatures un peu plus faibles $\left(18,3-19,5^{\circ} \mathrm{C}\right.$ contre $20,2-21,9^{\circ} \mathrm{C}$ à une humidité un peu plus élevée (65 - 75\% contre 53 $75 \%$ ) et à des éclairements moins intenses (maximum : 1300 contre 1500; minimum : 15 contre $50 \mu \mathrm{mol} \cdot \mathrm{m}^{-2} \cdot \mathrm{s}^{-1}$ ). Aucune de ces conditions ne peut être responsable des phénomènes constatés (fig 5) : dès les premières minutes, le potentiel des feuilles de la branche coupée chute bien au-dessous de celui des feuilles en place alors que la conductance n'est pas modifiée. La fermeture des stomates intervient plus tardivement, quand le potentiel foliaire atteint 1,9 à 2,4 MPa. En fin de journée, les stomates des feuilles en place se ferment et leur potentiel remonte; sur la branche coupée la fermeture est plus poussée pendant que le potentiel reste très bas.

\section{DISCUSSION}

Sur le site de la présente étude, la transpiration du frêne peut être bien supérieure à celle d'autres arbres tempérés (Tableau IV), que l'on considère les va- 


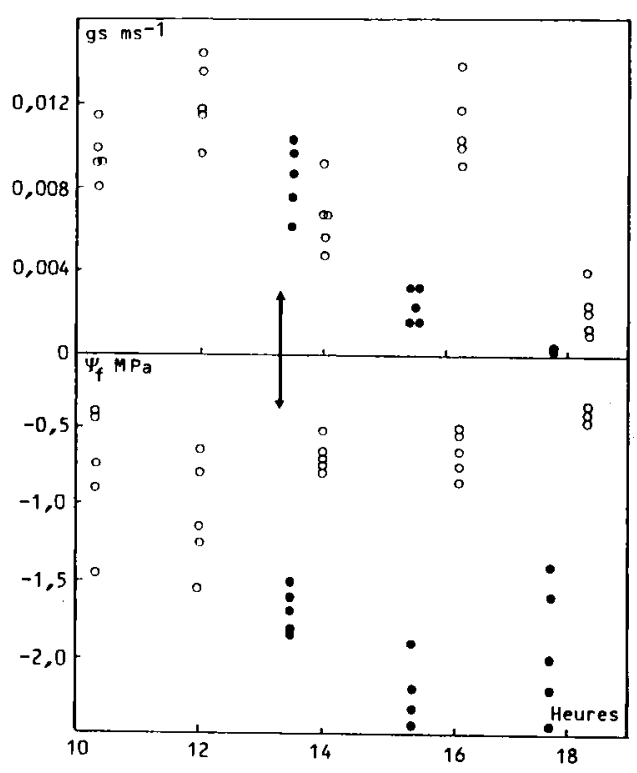

Fig 5. Évolution de la conductance (en haut) et du potentiel hydrique (en bas) de feuilles en place (signes ouverts) et de feuilles d'une branche coupée au moment indiqué par la double flèche (signes fermés). Journée du 27 juill 1987.

leurs instantanées maximales ou les estimations journalières (obtenues ici par intégration des courbes des figs 1 et 2). Ladefoged (1963) a obtenu des valeurs moindres que les nôtres mais, semble-t-il, dans un biotope différent. Le frêne en situation alluviale est bien gros consommateur d'eau.

Cette caractéristique est, pour une part, liée à la forte valeur de la conductance stomatique maximale : $16 \times 10^{-3}$ $\mathrm{m} \cdot \mathrm{s}^{-1}$. Par comparaison, les valeurs de la conductance maximale de toute une série d'espèces d'arbres, calculées d'après les résistances compilées par Körner et al (1979) ne dépassent pas $5 \times 10^{-3} \mathrm{~m} \cdot \mathrm{s}^{-1}$. Hinckley et al (1978) font état de 28 espèces dont la conductance maximale va de 2,2 a $4 \times 10^{-3}$ $\mathrm{m} \cdot \mathrm{s}^{-1}$. Celle de Fraxinus americana est estimée à $2,7 \times 10^{-3} \mathrm{~m} \cdot \mathrm{s}^{-1}$ (Davies et Kozlowski, 1974). En revanche, Besnard (1987) a trouvé $20 \times 10^{-3} \mathrm{~m} \cdot \mathrm{s}^{-1}$ comme valeur maximale pour la conductance de l'aulne blanc [Alnus incana (L) Moench] et $16 \times 10^{-3} \mathrm{~m} \cdot \mathrm{s}^{-1}$ pour celle du saule cendré (Salix cinerea $L$ ). Celle de certains peupliers atteint $14 \times 10^{-3} \mathrm{~m} \cdot \mathrm{s}^{-1}$ (Pallardy et Kozlowski, 1981).

D'après Hinckley et al (1978), la conductance maximale de beaucoup d'arbres est réalisée dès que l'éclairement dépasse $200 \mu \mathrm{mol} \cdot \mathrm{m}^{-2} \cdot \mathrm{s}^{-1}$; à cet éclairement le frêne n'atteint que la moitié de sa conductance maximale mais ce niveau est déjà bien supérieur à celui des conductances maximales citées plus haut.

L'éclairement est le facteur essentiel de l'ouverture stomatique du frêne dans les conditions du biotope étudié; les autres facteurs microclimatiques ne limitent cette ouverture que par temps sec et très chaud. De plus, le potentiel hydrique foliaire n'atteint jamais la valeur critique pour laquelle la fermeture est déclenchée. D'après Aussenac et Lévy (1983) ce potentiel critique est de $-3 \mathrm{MPa}$ pour des sujets de 2 ans en cuves de végétation. L'expérience du rameau coupé montre qu'une fermeture variable, mais pouvant être importante, se fait a partir de $-2 \mathrm{MPa}$, mais il est possible que les réactions des feuilles d'un tel rameau soient différentes de celles des feuilles sur pied (Tobiessen et Kana, 1974). Bien entendu le potentiel critique est un repère mais n'est probablement pas un facteur direct de la fermeture : Beadle et al (1978) rappellent que le fonctionnement des stomates est actionné par la turgescence des cellules gardiennes, laquelle peut avoir des relations variables avec $\psi_{f}$. Chez Nerium oleander Gollan et al (1985) montrent que la conductance 


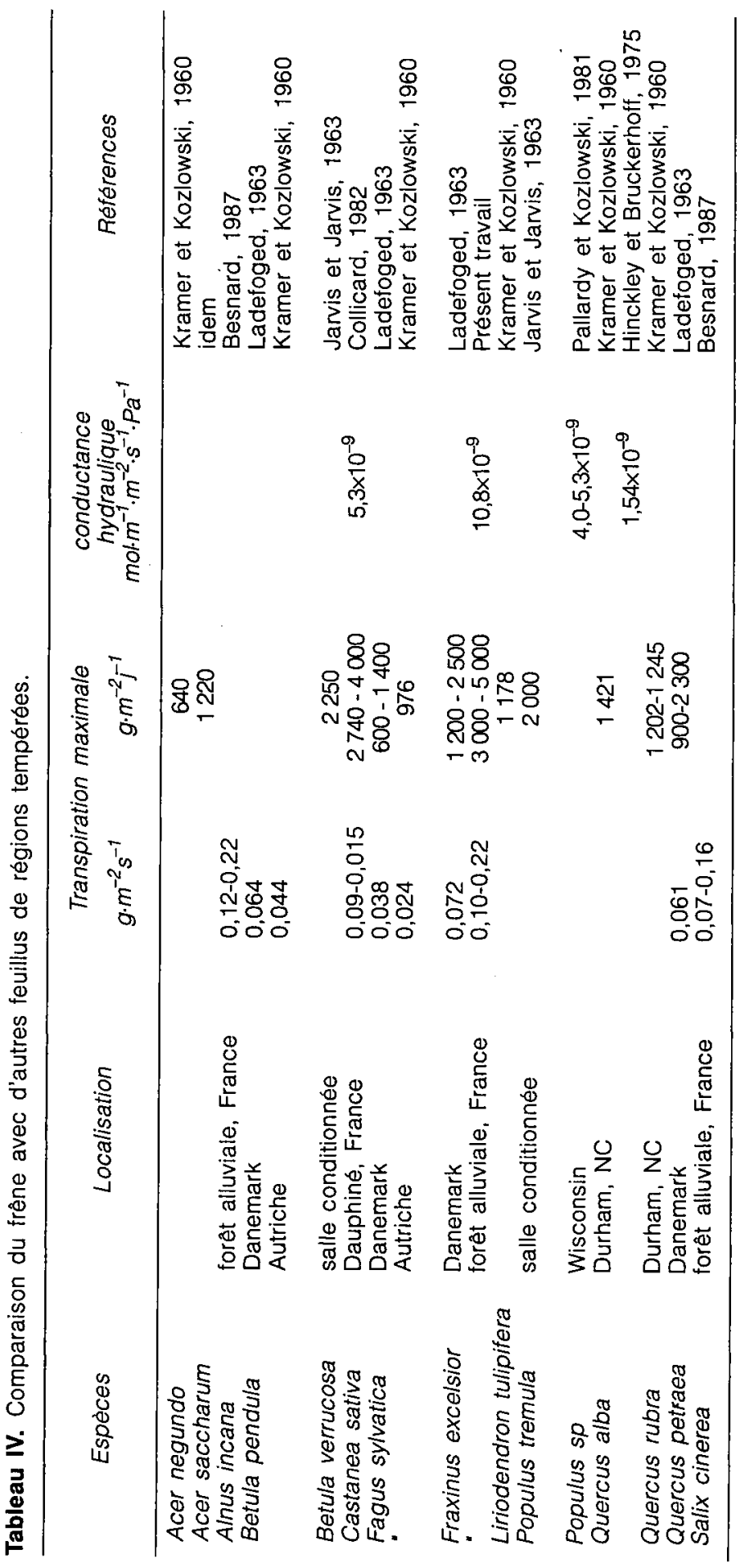


stomatique est en relation plus étroite avec l'état de l'eau dans le sol qu'avec le potentiel hydrique foliaire.

Nonobstant ces réserves, le potentiel critique de plusieurs espèces de feuillus a été déterminé : il est habituellement compris entre $-1,5$ et 2,5 MPa (Hinckley et al 1978). Celui du frêne se place dans le bas de cet intervalle, ou même au-delà, mais il n'est jamais atteint dans les conditions du biotope. En fait la caractéristique la plus remarquable du frêne est que son potentiel hydrique minimal est le même que celui de beaucoup d'autres arbres (voir, par exemple, Roberts et al 1979), alors que sa transpiration est intense. C'est ce qu'exprime la valeur de sa conductance hydraulique, qui excède celle d'autres feuillus (tableau IV) et, a fortiori de conifères (Schulze et al, 1985; Granier et Claustres, 1989). La conductance hydraulique dont il s'agit se rapporte au trajet du sol aux feuilles et prend donc en compte la conductance du sol. Or celle-ci est toujours très bonne grâce au taux élevé d'humidité (Daudet et Vachaud, 1977).

Sur un tel sol il est normal que l'évolution journalière du potentiel hydrique foliaire et celle de la conductance stomatique soient conformes aux modèles 1 ou 2 de Hinckley et al (1978), caractérisant des conditions non limitantes pour l'absorption de l'eau. Dans de telles conditions, Kaufmann (1977) vérifie que la relation du potentiel à la transpiration est linéaire et ne montre pas d'hysteresis. C'est bien ce qui est constaté ici. Pour Katerji et al (1983) ce type de relation dénote l'absence de contribution des réservoirs internes de l'arbre. II reste à vérifier chez le frêne en biotope sec que ce comportement est modifié en cas de sécheresse.

\section{CONCLUSION}

L'aptitude du frêne à transpirer intensément, en biotope alluvial a nappe phréatique peu profonde, s'explique par une conductance stomatique très élevée, proche de son maximum pendant une grande partie de la journée, tant que l'éclairement est supérieur à 700 $\mu \mathrm{mol} \cdot \mathrm{m}^{-2} \cdot \mathrm{s}^{-1}$. Même en cas de transpiration intense, le potentiel hydrique foliaire ne tombe pas au dessous de $-1,94 \mathrm{MPa}$ (en moyenne), ce qui témoigne d'une bonne conductance hydraulique. Le potentiel critique pour lequel les stomates se fermeraient n'est pas atteint, ce qui est probablement un élément important de la haute productivité des frênaies alluviales.

\section{REMERCIEMENTS}

Les auteurs remercient $\mathrm{M}$ le professeur Pautou pour les avoir fait bénéficier de sa connaissance des formations alluviales, $M$ Thony pour son aide dans l'étude de l'eau du sol, M Gruffaz, directeur, et les techniciens de l'Entente interdépartementale pour la démoustication, pour leur collaboration aux travaux de terrain.

\section{RÉFÉRENCES}

Aussenac G (1985) Le potentiel hydrique de l'arbre : une donnée essentielle pour la compréhension de l'écophysiologie des essences forestieres. Sci Sol, 1985217 226

Aussenac G, Levy G (1983) Influence du dessèchement du sol sur le comportement hydrique et la croissance du Chêne pédonculé (Quercus pedunculata Ehrl) et du Frêne (Fraxinus excelsior L) cultivés en cases de végétation. Ann Sci For 40, 251-264

Beadle CL, Jurner NC, Jarvis PG (1978) Critical water potential for stomatal clo- 
sure in Sitka Spruce. Physiol Pla 43, 160165

Besnard G (1987) Ecophysiologie de trois essences alluviales et contribution de la nappe phréatique à leur alimentation en eau dans les forêts riveraines du HautRhône français. Thèse Doct Univ Scient méd Grenoble, $124 \mathrm{p}$ et 29 annexes

. Besnard G, Carlier G (1988) Le régime hydrique de l'Aulne blanc (Alnus incana ( $L$ ) Moench) dans une forêt riveraine du Haut-Rhône. Bull Écol 19, 1-11

Braun HJ (1977) Growth and water economy of the trees Acer platanoides $\mathrm{L}$ Acer pseudoplatanus $\mathrm{L}$ and Fraxinus excelsior L. Z Planzenphysiol 84 459-462

Burrows FJ, Milthorpe FL (1976) Stomatal conductance in the control of gas exchange, In Water deficits and plant growth (Kozlowskit T, ed) Acad Press, 4, 103-152

Collicard JJ (1982) Étude écophysiologique des taillis de châtaigniers (Castanea sativa Miller) du Bas-Dauphiné en 1981 et 1982 ; potentiel hydrique, résistance à la diffusion et transpiration des feuilles. Thèse $3^{e}$ cycle, Univ scient médic Grenoble, $42 \mathrm{p}$

Daudet FA, Vachaud G (1977) La mesure neutronique du stock d'eau du sol et ses variations. Application à la détermination du bilan hyárique. Ann Agron, 27, 165182

Davies WJ, Kozlowski TT (1974) Stomatal responses of five woody angiosperms to light intensity and humidity. Can J Bot 52 , 1525-1534

Devauchelle R, Lévy G (1977) Proprietés stationnelles et croissance du frêne dans l'Est de la France, étude de certaines caractéristiques de cette essence. Ann Sci For 34, 231-244

Elfving DC, Kaufmann MR, Hall AE (1972) Interpreting leaf water potential measurements with a model of the soil-plant-atmosphere continuum. Physiol Plant 27, 161-168

Gollan T, Turner NC, Schulze ED (1985) The response of stomata and leaf gas exchange to vapor pressure deficits and soil water content. 3 . In the sclerophyllous woody species Nerium oleander. Ecologia 65, 356-362

Granier A, Claustres JP (1989) Relations hydriques dans un épicea (Picea Abies L) en conditions naturelles : variations spatiales. Acta Oecol Oecol Plant 10, 295310

Hinckley TM, Bruckerhoff DN, 1975. The effects of drought on water relations and stem shrinkage of Quercus alba. Can $J$ Bot 53, 62-72

Hinckley TM, Lassoie JP, Running SW (1978) Temporal and spatial variations in the water status of forest trees. For Sci 24 , Monograph 20, $79 \mathrm{p}$

Jarvis PG, Jarvis MS (1963) The water relations of tree seedlings. I. Growth and water use in relation to soil water potential. Physiol Plant, 16, 215-235

Katerii N, Hallaire $M$, Perrier A, Durand $R$ (1983) Transfert hydrique dans le végétal. I Modélisation à l'échelle du couvert végétal en conditions naturelles. Acta Oecol Oecol Plant 4 (1), 11-26

Kaufmann MR (1977) Soil temperature and drying cycle effects on water relations of Pinus radiata. Can J Bot 55, 2413-2418

Körner Ch, Cochrane PM (1985) Stomatal responses and water relations of Eucalyptus pauciflora along an elevational gradient. CEcologia 6, 443-455

Körner Ch, Schell JA, Bauer H (1979) Maximum leaf diffusive conductance in vascular plants. Photosynthetica 13, 45-82

Kramer P, Kozlowski TT (1960) The Physiology of trees, McGraw-Hill, New York, $642 \mathrm{pp}$

Ladefoged L (1963) Transpiration of forest trees in closed stands. Physiol Plant 16, 378-414

Le Goff N, Lévy G (1984) Productivité du frêne (Fraxinus excelsior $L$ ) en région Nord-Picardie B. Etude des relations entre la productivité et les conditions de milieu. Ann Sci For 41, 135-170

Milburn JA (1979) Water flow in plants. Longman, London $225 \mathrm{p}$

Pallardy SG, Kozlowski TT (1981) Water relations of Populus clones. Ecology 62 159-169

Pautou G, Decamps H (1985) Ecological interactions between the alluvial forests and hydrology of the upper Rhone. Acta Hydrobiol 104, 13-37

Richard L, Pautou G (1982) Carte de la végétation de la France au $200000^{\circ}$. Alpes du Nord et Jura méridional. Notice détaillee des feuillesd 48 Annecy - 54 
Grenoble. Éditions du CNRS, Paris 317 $p$

Roberts SW, Knoerr KR, Strain BR (1979) Comparative field water relations of four co-occuring forest tree species. Can $J$ Bot 57, 1876-1882

Scholander PF, Hammel HT, Bradstreet ED, Hemmingsen EA (1965) Sap pressure in vascular plants. Science 148, 339-346

Schulze ED, Cermak J, Matyssek R, Penka $M$, Zimmermann $R$, Vasicek $F$, Gries $W$, Kucera J (1985) Canopy transpirations and water fluxes in the xylem of the trunk of Larix and Picea trees. A comparaison of xylem flow, porometer and cuvette measurements. Oecologia 66, 475-483

Tobiessen P, Tood KM (1974) Drought stress avoidance in three pioneer trees species. Ecology 55, 667-670

Vachaud G, Dancette C, Sonko S, Thony JL (1978) Methodes de caractérisation hydrodynamique in situ d'un sol non saturé. Application à deux types de sols au Sénégal en vue de la détermination des termes du bilan hydrique. Ann agron 29, $1-36$ 